\title{
Quantum Clocks with Double Audit and Relations with Gravity Harsh Jindal ${ }^{1}$, Jagdeep Kaur ${ }^{*}$ \\ ${ }^{1}$ Department of Computer Science and Engineering, Chandigarh University, Punjab, India ${ }^{1}$ \\ ${ }^{2}$ Department of Physics, Chandigarh University, Punjab, India ${ }^{1^{*}}$
}

Article Info

Volume 8 Issue 2

Page Number: 333-336

Publication Issue :

March-April-2021

Article History

Accepted : 15 April 2021

Published : 24 April 2021

\section{ABSTRACT}

In this paper, a non-repeating quantum algorithm is introduced that depends on detectable Byzantine's quantum solution that attains Clk synchronization in the arbitrary faultier processes' presence through utilizing a double quantum system only. Precisely, it has been shown that general relativistic mass-energy equivalences intimate gravitational relation among Clks, however, energy's quantum mechanical superposition resulted in a non-fixed metric background. Depending only on assumptions that gravitational principles held in such conditions, it has been shown that the Clks essentially gets involve over time dilation effect that ultimately results in a double Clk coherence loss. Therefore, the measured time through a double Clk is not defined precisely. Although, the time's general relativistic notion is recuperated in the Clks conventional limits.

Keywords - Relativistic Mass-energy, Gravity, Double Audit, Time, Quantum Mechanics.

\section{Abbreviation \& Symbol}

\section{INTRODUCTION}

- Sync-Synchronization

- $\mathrm{Clk}$ - Clock

- ICA - Interactive Consistency Algorithm

- DBA - Detectable Byzantine Algorithm.

- $\varphi 0 / \psi$ in - Initial Work function

- $\psi$ - Final Work Function

- $\Delta \mathrm{E}$ - Change in Energy

- G - Gravitation

- $\boldsymbol{h}$ - Reduced Planks Constant
In general relativity's context, time is locally specified in proper time terms along world lines. This is affirmed that along these world lines Clks correspond to metric fields in a manner that theory of "Clk Hypothesis" predicted proper time and their readings were coincided. Further, following tasks are attained by the Clk synchronization algorithm.

In a particular time, every non-faulty processes time, there must be similar Clks. It is needed however not ample as in simple terms every Clk is stopped at zero assures completion of process. Moreover, further 
errors might be resulted due to synchronizing therefore, we need that. On the amount, there exists a small bound that during synchronization a process Clk is altered. Genuine Clk Sync algorithm might be complex. For simplifying the issue, following assumptions were utilized for working:

- At first, every Clk is Sync to alike values. Usually perfect time is not possessed by physical Clks however, they drifted to respect each other.

It inspires the subsequent assumptions:

- Each non-faulty process Clks in real time runs at 1 second in Clk time/second. A normal issue come about from the Clks constantly switching throughout Sync procedures. The issues will be caused, due to the fast Sync algorithm. It stimulates the final assumption:

- $\quad$ The time difference among the other process Clk and non-faulty process Clk is read by nonfaulty process. ICA is utilized as a method for achieving the Sync where each non-faulty process resulted in a mutual agreement related to every Clk. It is required that for each process P1 it must be satisfied by an ICA. Any 2 nonfaulty process acquire process p's Clk's similar values, even when there exists faults. 2 . In case this is non-faulty, then each non-faulty process attains the p's Clk quit values. The conditions for ICA make them suitable for the project of sympathetic tolerant Sync. For highest applications it is enough to contemplate a situation known as DBA. In this case, it is required that: 1 . either all non-faulty process attain the similar values or every process must abort, as well as 2 . In case process $\mathrm{p}$ is nonfaulty, at that time either each non-faulty process attains similar values or aborts.

\section{HYPOTHESIS USED}

A reference frame's common picture in space time utilizes Clks' lattice work for locating events. A spectator, along with a space time's specific split in space as well as time, points Clk locally, to a space region. The spectator then looks through the data noted by the Clks at his/her location. As a normal characteristic, a quantum $\mathrm{Clk}$ is described as a system in an energy eigen states emplacement. In such a manner, it has been shown that how time dilation's general (classical) relativistic notion appears in terms of gravitating quantum system's average mass energy from our model.

\section{MODEL FOR CLK:}

Let there be 2 gravitationally interacting Clks, labelled as $\mathrm{AA}$ and $\mathrm{BB}$, and a coordinate distance $\mathrm{xx}$ (in far-away observer frame) is utilized for their separation. For a minimum estimation to the Einstein equations explication, the Newtonian gravitational energy describes the gravitational interaction as

$$
U(x)=-G m A m B / x U(x)=-G m A m B / x .
$$

Here, only first-order estimation is targeted for the metric explication. Although, in the same manner, post-Newtonian corrections can be considered. Through such masses, the complete contribution of mass energy to the gravitational field, containing both the dynamical mass and static rest mass $\mathrm{mm}$ which corresponds to freedom's internal degrees energy $\mathrm{H}^{\text {int }} \mathrm{H}^{\text {int. }}$. The dynamical mass notion having unblended relativistic nature as well as come nearly from our composite particle's constituents' interaction.

Actually, from a relativistic viewpoint, logically, there does not exists any differences among interaction energy and mass, as well as the difference among them is effectively energy scale matter through that system is inquested. For expressing the interactivity in terms of quantum 
mechanical through each particle mass elevation to operators as well as utilizing the mass-energy equivalence:

$$
m \rightarrow m+H^{\wedge} \text { int } / c 2 m \rightarrow m+H^{\wedge} \text { int } / c 2
$$

For modesty, we suppose that in comparison to dynamical one, static mass is slightly smaller as well as emphasize on consequence only because of the freedom's internal degrees. Therefore, for the two-Clk system, the Hamiltonian is

$$
\begin{gathered}
H^{\wedge}=H^{\wedge} A+H^{\wedge} B-G c 4 x H^{\wedge} A H^{\wedge} B \cdot H^{\wedge}=H^{\wedge} A+H^{\wedge} \\
B-G c 4 x H^{\wedge} A H^{\wedge} B .
\end{gathered}
$$

Consider that both Hamiltonians $\mathrm{H}^{\wedge} \mathrm{AH} \mathrm{H}^{\wedge} \mathrm{A}$ and $\mathrm{H}^{\wedge} \mathrm{BH} \mathrm{H}^{\wedge} \mathrm{B}$ energies are same as well as there exists uncorrelated Clks initial states as:

$$
\mid \psi \text { in }\rangle=[(|0\rangle+|1\rangle) / 2-\sqrt{ }] \otimes 2 / \psi \text { in }\rangle=[(|0\rangle+|1\rangle) / 2]
$$

As per the far-away observer, the state at time is:

$$
\begin{gathered}
|\psi\rangle=12-\sqrt{ }(|0\rangle)(\varphi 0\rangle+e-i t \hbar \Delta E / 1\rangle / \varphi 1\rangle), \\
|\psi\rangle=12(|0\rangle / \varphi 0\rangle+e-i t \hbar \Delta E / 1\rangle / \varphi 1\rangle),
\end{gathered}
$$

Where

$$
\begin{aligned}
& |\varphi 0\rangle=(|0\rangle+e-i t \hbar \Delta E(1\rangle) / 2-\sqrt{ }|\varphi 0\rangle=(|0\rangle+e-i t \hbar \Delta E / 1\rangle) / 2, \\
& \text { and } \\
& |\varphi 1\rangle=[|0\rangle+e-i t \Delta E \hbar(1-G \Delta E c 4 x) / 1\rangle] / 2- \\
& \sqrt{ }|\varphi 1\rangle=[|0\rangle+e-i t \Delta E \hbar(1-G \Delta E c 4 x) / 1\rangle] / 2 \text {. }
\end{aligned}
$$

It has been observed that with the gravitational interaction Clks get entangled. The time rate running in one Clk is related with the other Clk's energy value. Since tmix-tmix is approached, any Clk's reduced state approach the maximum mixed state as well as Clk does not having ability for functioning as a proper Clk due to the reason that in cases only arbitrary answers are acquired when Clk is asked for the time. Further, the tmix-tmix values are not altered by the static mass presence in the Hamiltonian, since it doesn't entering in
Hamiltonian's any part which contributed to Clks entanglement.

This is significant figuring out that for such effect to come about, this is essential that Clks internal energy is considered as a quantum operator, instead of only considering the energy's expectation value, as performed in semi-classical gravity. Labels AA and $\mathrm{BB}$ interchanged. After that it has been shown that Clks don't get interwine, in the semiclassical approach, as well as the result is one Clk's overall time dilation because of the other Clk mean energy.

The particular effect fundamentally affects the time measurements which were followed by general relativity in the weak-field limit as well as from quantum mechanics. It does not depends on the usual argument that concerns space-time intervals measurability limitations because of formation of black hole.

\section{RESULT}

The Paper consist of Derived constraints of Clk Sync $|\varphi 1\rangle=[|0\rangle+e-i t \Delta E \hbar(1-G \Delta E c 4 x) \quad|1\rangle] / 2-$ $\left.\sqrt{ }|\varphi 1\rangle=\left[|0\rangle+e-i t \Delta E \hbar\left(1-G \Delta E_{c} 4 x\right) / 1\right\rangle\right] / 2$.

The Hypothesis used here are already used in many researches and inventions

The paper discuss the defects present while doing or combining two atoms during Clk Sync process, the paper also discusses its relation with gravity on a large scale .

\section{DISCUSSION}

This resolves DBA as well as Clk synchronization is achieved in arbitrary various faulty Clks presence. These Clks spot world lines as well as pulse as per the metric tensor values along their trajectory. 


\section{CONCLUSION}

Besides giving some unrealistic values the optimization approach proves to be a breakthrough in solving complex nonlinear constrained problems with a very good accuracy. However, the results were in accordance with the general rule of Clk Sync and can lead to improved results with removal of various assumptions.

\section{REFERENCES}

[1]. Lock, M. P. E., Fuentes, I., Renner, R. \& Stupar, S. Time in Physics, Tutorials, Schools, and Workshops in the Mathematical Sciences 51-68 (Springer, Cham,2017).

[2]. Ruiz, E. C., Giacomini, F. \& Brukner, Č. Entanglement of quantum Clks through gravity. PNAS 114, E2303-E2309 (2017).

[3]. Zych, M., Rudnicki, Ł. \& Pikovski, I. Gravitational mass of composite systems. Phys. Rev. D 99, 104029 (2019).

[4]. Paige, A. J., Plato, A. D. K. \& Kim, M. S. Classical and nonclassical time dilation for Quantum Clks. Phys. Rev. Lett. 124, 160602 (2020).

[5]. Lock, M. P. E. \& Fuentes, I. Quantum and classical effects in a light-Clk falling in Schwarzschild geometry. Class. Quantum Grav. 36, 175007 (2019).

[6]. R. Jozsa, D. S. Abrams, J. P. Dowling, and C. P. Williams, Phys. Rev. Lett. 87, 129802 (2001).

[7]. M. Krco and P. Paul, Phys. Rev. A 66, 024305 (2002).

[8]. R. Ben-Av and I. Exman, Phys. Rev. A 84, 014301 (2011).

[9]. C. Ren and H. F. Hofmann, Phys. Rev. A 86, 014301 (2012).

\section{Cite this article as :}

Harsh Jindal, Jagdeep Kaur, "Quantum Clocks with Double Audit and Relations with Gravity", International Journal of Scientific Research in Science, Engineering and Technology (IJSRSET), Online ISSN : 2394-4099, Print ISSN : 2395-1990, Volume 8 Issue 2, pp. 333-336, March-April 2021. Available at doi : https://doi.org/10.32628/IJSRSET218276 Journal URL : https://ijsrset.com/IJSRSET218276 